\title{
The Effects of Experimentally Induced Rumination, Positive Reappraisal, Acceptance, and Distancing When Thinking About a Stressful Event on Affect States in Adolescents
}

\author{
Lea Rood • Jeffrey Roelofs • Susan M. Bögels • \\ Arnoud Arntz
}

Published online: 26 July 2011

(C) The Author(s) 2011. This article is published with open access at Springerlink.com

\begin{abstract}
The current study compares the effects of experimentally induced rumination, positive reappraisal, distancing, and acceptance on affect states in adolescents aged 13-18. Participants $(N=160)$ were instructed to think about a recent stressful event. Next, they received specific instructions on how to think about that event in each condition. Manipulation checks revealed that the manipulations were successful, except for acceptance. The two most reported events were "a fight" and "death of loved one". Results showed that positive reappraisal (i.e., thinking about the benefits and personal growth) caused a significantly larger increase in positive affect and decrease in negative affect compared to rumination, distancing, and acceptance. Current findings implicate that positive reappraisal seems an adequate coping strategy in the short-term, and therefore could be applied in interventions for youth experiencing difficulties managing negative affect. Future research should focus on long-term effects of these cognitive strategies and on more intensive training of acceptance.
\end{abstract}

Keywords Acceptance $\cdot$ Adolescents $\cdot$ Distancing · Positive reappraisal $\cdot$ Rumination

L. Rood $(\bowtie) \cdot$ J. Roelofs $\cdot$ A. Arntz

Dept. of Clinical Psychological Science, Maastricht University, P.O. Box 616, 6200 MD Maastricht, The Netherlands

e-mail: J.Roelofs@maastrichtuniversity.nl

S. M. Bögels

Research Institute of Child Development and Education,

University of Amsterdam,

Amsterdam, The Netherlands

\section{Introduction}

Understanding how various cognitive strategies in response to stress impact affective states among adolescents is important, as it may shape intervention or prevention strategies for adolescents who are experiencing difficulties with managing negative affect related to personal stress (Compas et al. 2001). Various cognitive coping strategies have been described in the literature. The present study focuses on rumination, positive reappraisal, acceptance, and distancing.

Rumination can be defined as repetitively thinking about the causes, implications, and consequences of negative feelings (Nolen-Hoeksema 1987, 1991) and stressful events (Robinson and Alloy 2003). Although people ruminate in an attempt to gain insight in their problems (Nolen-Hoeksema 1991), rumination is likely to have unconstructive consequences because of its abstractanalytical nature, negative content, and its likelihood to occur in negative intrapersonal and situational context (Watkins 2008). Cross-sectional studies have demonstrated that rumination is strongly associated with symptoms of depression and anxiety in adults as well as in adolescents (see reviews Nolen-Hoeksema et al. 2008; Rood et al. 2009; Watkins 2008). Prospective studies have demonstrated that rumination predicts depression in adults (Nolen-Hoeksema et al. 2008; Watkins 2008), and changes in depressive symptoms over time in youth (Rood et al. 2009). Only one study so far has examined the effect of rumination on depressive mood state in an adolescent sample experimentally. Park et al. (2004) compared the effects of an 8-minute induction of self-focused rumination (i.e., a self-focus on emotions, body state, goals and attributes) to distraction in adolescents aged 12-17. Their 
findings showed that rumination as compared to distraction worsened depressive mood in clinically depressed adolescents and in community controls.

Positive reappraisal ${ }^{1}$ is a meaning-based type of coping that can be defined as reinterpreting events or situations in a positive manner (Folkman and Moskowitz 2000; Helgeson et al. 2006), and includes elements such as attempting to find benefits and personal growth (see conceptualisation of Garnefski et al. 2001). Positive reappraisal of stressful experiences increases awareness of important personal values in life, and encourages to act upon those values (Folkman and Moskowitz 2000), thereby changing the meaning of stressful experiences. As a cognitive coping strategy in response to stressful events, positive reappraisal has been positively related to psychological wellbeing, and to a lesser extent, negatively related to depressive symptoms in adults (see review Helgeson et al. 2006), as well as in adolescents (Garnefski et al. 2001; Garnefski et al. 2002). Tugade and Fredrickson (2004) have suggested that especially people who have a low resiliency to stress might benefit from a training in the use of positive reappraisal, as it generates positive affect and facilitates adequate emotion regulation. Folkman (2008) stresses that the experience of positive emotions can play an important adaptive role in coping with stress, for example, by influencing the type of coping strategy that is employed, but also by "charging up the batteries". An elaborated theory on the role of positive emotions is provided by Fredrickson (2004), whose broaden-and-build theory posits that positive emotions generate a broader mindset (i.e., momentary thoughtaction repertoire), which can lead to an increase (build) in physical, social, psychological, and intellectual resources that later help form a buffer against stress. In the current study, the positive reappraisal condition aimed to induce a thinking process focused on benefits and personal growth as a consequence of the stressful event.

Another cognitive coping strategy which could be considered an adaptive alternative to rumination, is mindfulness-based acceptance of thoughts and feelings. Acceptance is one of the core elements of mindfulness (Germer 2005; Williams et al. 2007). The purpose of acceptance in mindfulness practice is not to passively

\footnotetext{
${ }^{1}$ Please note that positive reappraisal should not be confused with James Gross' cognitive reappraisal, which he defines as "changing how we think about a situation in order to decrease its emotional impact" (p. 281, Gross 2002). Although positive reappraisal and cognitive reappraisal seem similar cognitive processes in the sense that they aim to change interpretations and appraisals into more positive ones, in the current study design, positive reappraisal is considered a post-event coping strategy, and not an antecedent emotion-focused regulation strategy as conceptualised by Gross (2002).
}

resign to unwanted experiences or emotions, but rather to become aware of thoughts and feelings, observe them as they are, and to accept their presence without judging them as good or bad (Baer 2003; Williams et al. 2007). Mindfulness-based approaches have yielded promising results with regard to the treatment of a wide range of psychological problems in adults, such as dealing with chronic pain, depression, anxiety, and ADHD (Baer 2003). The effects of mindfulness approaches have not yet been extensively studied in youth samples (see Burke 2010); however, intervention studies so far show promising results in adolescents with a range of psychiatric disorders (Biegel et al. 2009; Bögels et al. 2008).

Experimental studies have demonstrated that mindfulness meditation (or mindful acceptance; Singer and Dobson 2007) was significantly more effective in recovering from a sad mood induction than rumination in undergraduate students (Broderick 2005) and in remitted and currently depressed adults (Huffziger and Kuehner 2009; Singer and Dobson 2007). In contrast to these studies, Kuehner et al. (2009) found that mindfulness meditation did not have a beneficial effect on mood compared to rumination in a sample of undergraduate students. In sum, there is evidence that mindful self-focus or acceptance might be more effective than rumination when processing stressful experiences, though results so far are equivocal. In the present study, the acceptance condition was aimed to induce an attentional, experiential focus on thoughts about the stressful event.

Self-distancing (i.e., looking at self from an observer's perspective) has a beneficial effect on level of depression (Kross and Ayduk 2009) and may prevent rumination (Kross et al. 2005). An observer's perspective may reduce emotional arousal when working through negative events, by looking more objectively at the circumstances (Kross et al. 2005; Nigro and Neisser 1983). However, the observer's perspective also plays a role in the maintenance of certain psychological disorders: as a maladaptive attentional process in social anxiety (see Bögels and Mansell 2004) and as a cognitive avoidance strategy in PTSD (Kenny and Bryant 2007). A distancing induction was included with the intention to make participants think about all negative aspects of the stressful event from an observer's perspective, thus in a non-ruminative (and non-verbal) manner. A neutral control condition was not included in our study, as a "think about anything" manipulation without instructions would allow participants to employ various types of coping strategies including the ones we wanted to manipulate in the other conditions (such as rumination), and would therefore not adequately serve as a control condition.

Whereas cross-sectional and longitudinal studies provide information on the strengths of relationships between variables, experimental studies enable a more direct 
examination of causal relationships. The purpose of this study was to compare the effects of various cognitive coping strategies i.e., rumination, positive reappraisal, acceptance, and distancing, on positive and negative affect states related to personal stress in a sample of non-clinical adolescents. We expected that (1) participants would show a greater increase in negative affect after the rumination induction compared to the acceptance, positive reappraisal, and distancing inductions; and (2) participants would show a stronger increase in positive affect, and a stronger decrease in negative affect, after the positive reappraisal induction compared to the other inductions. As the consequences of rumination have been found to be more unconstructive in the presence of other vulnerability factors such as low self-esteem and negative beliefs (see Watkins 2008), we examined whether the effect of condition on affect states would be moderated by the level of trait-rumination (SRRS-C; see Measures). High traitruminators were expected to report a greater increase in negative affect after the rumination induction compared to low trait-ruminators. More speculatively, high traitruminators were expected to respond less favourably to the expected relatively beneficial effects of the acceptance and positive reappraisal manipulations, compared to the low trait-ruminators.

Additional hypotheses were formulated on differences between conditions regarding certain characteristics that may co-determine the outcome of cognitive strategies. Intrinsic to meaning-based coping, rumination and positive reappraisal are both abstract styles of thinking (Folkman 2008; Watkins 2008). There is some evidence that the beneficial effects of mindfulness are due to the reduction of abstract-evaluative thinking (Heeren and Philippot 2009). Therefore, the level of abstractness of thoughts was expected to be higher in the rumination and positive reappraisal conditions than in the acceptance and distancing conditions. As abstractness of thought may be related to a predominance of words over images in thought (see Stöber and Borkovec 2002), the ratio of words by images was expected to be higher in the rumination and positive reappraisal conditions than in the acceptance and distancing conditions. The level of concreteness of thoughts was expected to be highest in the distancing condition, which is likely to evoke imagery because of its visual focus. Furthermore, although a component of rumination, self-focused attention is not necessarily maladaptive. Self-focus in a positive intrapersonal and environmental context has been found to reduce negative affect (see Mor and Winquist 2002). There is some evidence that mindful self-focus improves sad mood (Huffziger and Kuehner 2009). The degree of self-focus was expected to be similar across conditions.

To the authors' best knowledge, the present study is the first study to compare the short-term effects of rumination, positive reappraisal, acceptance, and distancing on negative and positive affect states in adolescents in an experimentally controlled design. The examination of how different strategies to cope with stressful experiences influence negative and positive affect states in non-clinical adolescents may generate hypotheses regarding maintaining or exacerbating variables in depression, which is characterized by the combination of high negative affect and low positive affect (Watson et al. 1988).

\section{Method}

\section{Participants}

The study sample consisted of 160 non-clinical adolescents aged 13 to $18(M=14.45, S D=1.10)$, of which 79 boys $(49.4 \%)$ and 81 girls. All levels of education were represented: lower professional/general secondary education $(N=76)$, higher general secondary education $(N=42)$, and pre-university education $(N=42)$. The nationality of the participants was predominantly Dutch $(96.9 \%)$. About one quarter of the participants $(24.4 \%)$ reported that their parents were divorced. Mean occupational level of both parents ${ }^{2}$ served as an indication of socio-economic status (SES): $27.5 \%, 36.9 \%$ and $29.4 \%$ of the participants came from families with a respectively low, average, and high socio-economic background; whereas of $6.3 \%$ of the participants, both parents were unemployed. Mean age did not differ across groups, $F(3,156)=0.11, p=0.96 . X^{2}$-tests showed that the four groups did not differ on demographic variables such as gender, educational level, nationality, divorce of parents, and SES (statistics obtainable from first author). There were two exclusion criteria: (1) recently being diagnosed with a mood disorder, for ethical reasons $(N=0)$, and (2) not being able to come up with a recent stressful event. This was checked verbally by the researcher prior to the manipulation of cognitive coping strategy $(N=2)$.

\section{Recruitment Procedure}

The participants were recruited at a secondary school in the southern region of the Netherlands. In accordance with the

\footnotetext{
${ }^{2}$ Occupational level of both parents was assessed by means of the Standard Classification of Occupations 1992: Edition 2001, as provided by the Dutch Central Bureau of Statistics, and was based on occupation of parent as reported by the participant. Mean occupational level was rounded up, so that in case mother's occupational level was low, while father's occupational level was average, then mean occupational level of family is average.
} 
school policy regarding the different educational levels involved, either the class mentors or the research assistant held a 5-minute talk to motivate the pupils to participate, and subsequently distributed information forms for parents and an adjusted version for adolescents. The pupils were not fully informed about the pupose of the study. Active written consent was obtained from participating adolescents and their parents. About 17.7\% $(N=275)$ of the approached pupils $(N=1552)$ and their parents agreed to participate. In order to have educational level, age, gender equally represented in the conditions, 160 pupils were effectively contacted, scheduled and eventually participated in the experiment. With regard to the selection of participants (i.e., stratification by gender, age, and educational level), the procedure was as follows: informed consents were randomly sorted by gender, age, and educational level, as the research assistant did not have further background information on the participants. For example, a group of girls aged 13-14 in the lower educational level were scheduled and subsequently randomly assigned to one of the conditions. These procedures are not likely to have led to a systematic bias or reduced generalizability in the final sample. The experimental protocol was approved by a local Institutional Review Board.

\section{Design}

The study has a between-subjects design with four experimental conditions: rumination $(N=40)$, acceptance $(N=40)$, positive reappraisal $(N=41)$, and distancing $(N=39)$. A repeated measures within subjects approach was applied to asses positive and negative affect states at four time points. The baseline measurement (T1) took place at the start of the experiment before the stressinduction; the second measurement (T2) after the stressinduction and before the manipulation of coping strategy; and the third measurement (T3) after the manipulation of coping strategy. A fourth measurement (T4) of affect states took place after the positive distraction task at the end of the experiment.

\section{Study Procedure}

The research assistant tested all participating pupils during regular classes, in a private test room at school. First, participants completed self-report measures of the disposition to ruminate (SRRS-C) and depressive symptoms (CDI; see "Measures"). Then baseline affect states were assessed by means of visual analogue scales (VAS; T1, see "Measures"). In all conditions adolescents were asked to think about a current or recent stressful experience that was troubling them for about $3 \mathrm{~min}$. They were specifically instructed to play the event in their head as a movie, and to bring up all thoughts and feelings that belonged to the event until they would see the event vividly before them and felt like they were in the middle of it again. Next, they were instructed to stop the movie at the most stressful moment, and to tell the researcher which stressful event had occurred to them. The full instructions of the stress-induction were given by vocal communication read aloud and on paper.

Then affect states were assessed again by VAS (T2). Subsequently, in order to manipulate the way of thinking about the stressful event, participants received instructions by vocal communication read aloud and using a set of four cards, specific to each condition. Participants were instructed to think about the stressful experience again, but now in a certain way, and to use the cards as a support to keep thinking the intended way. In the Appendix the instructions of the manipulations of cognitive coping strategy are given. The participant was left alone for $5 \mathrm{~min}$. After the experimental manipulation, a third series of VAS assessing affect states were administered (T3). To assess whether, and to what extent, the manipulation of cognitive coping strategies had succeeded, we relied on a qualitative (administered first) as well as quantitative manipulation check (see "Qualitative Manipulation Check (Observer-Rated VAS)" and "Quantitative Manipulation Check (Self-Rated VAS)"). At the end of the experiment, for ethical reasons participants were offered a positive mood induction task that consisted of listening to, and talking about, a favourite piece of music they had been instructed to bring to the experiment beforehand. The researcher checked by a fourth series of VAS (T4) whether participant's affect had restored to baseline level. The entire procedure took $45 \mathrm{~min}$. Participants received a gift voucher worth $\mathrm{E5}$ for their participation.

\section{Measures}

\section{Stress-Reactive Rumination}

The Stress-Reactive Rumination Scale for children (SRRS-C) is a downward extension of the SRRS for adults, adapted to youth (Robinson 1997; authorized Dutch version: Rood et al. 2010). The SRRS-C aims to measure the extent to which negative inferences following stressful events are rehearsed. The SRRS-C consists of nine items (e.g., "I think about how the stressful event was totally my fault"). The frequency of each item is rated on a four-point Likert type scale (i.e., 1=almost never, $2=$ sometimes, $3=$ often, $4=$ almost all the time). Reliability of the SRRS-C is adequate; discriminative validity and concurrent criterion validity are supported (Rood et al. 2010). 


\section{Depressive Symptoms}

The Children's Depression Inventory (CDI; Kovacs 1981; Dutch version: Timbremont and Braet 2001; 2002) aims to measure the level of depressive symptoms in children from 7 to 17 years old. It consists of 27 items, covering feelings of sadness, self-guilt, loss of appetite, insomnia, and adjustment to school. For each item, the subject chooses one of three statements (e.g., I am sad sometimes/I am often sad/I am always sad) that represents best how he or she has been feeling the last two weeks. Reliability in terms of internal consistency is good and the convergent validity of the CDI is supported (Timbremont and Braet 2001).

\section{Measurement of Affect States (VAS)}

Negative and positive affect states (i.e., "gloomy", "sad", and "happy") were assessed by means of three VAS, e.g., "How gloomy do you feel at the moment?", using a horizontal line $(0-100 \mathrm{~mm})$, with at the left end of the continuum "not at all" (0), and at the right end "very much" (100).

\section{Qualitative Manipulation Check (Observer-Rated VAS)}

First, participants were asked to describe in just a few words what stressful event(s) they had thought of, and to rate the severity of the event(s) on a five point Likert scale ("How bad was this event to you?" $1=$ not bad [not terrible $]$... $5=$ the worst I have ever experienced). Second, they were asked to describe what thoughts (words and images) exactly went through their mind during the experimental task, and to be as complete as possible in their descriptions. The resulting qualitative data in the form of short texts were rated by two independent observers (bachelor students) who were blind to condition. The observers rated the content of the texts as a whole with respect to the degree of rumination, positive reappraisal, distancing, and acceptance on VAS ("Indicate the degree to which the text fits the [rumination/distancing/ positive reappraisal/acceptance] condition according to you") using a horizontal line $(0-100 \mathrm{~mm})$, with at the left end of the continuum " $0 \%$ ", and at the right end " $100 \% "$. They were allowed to rate the content of the text on more than one coping strategy if that seemed appropriate. Furthermore, the written texts were rated on the degree of self-focus, concreteness, and abstractness on VAS ("To what extent is the subject focused on himself/herself?", "Indicate the degree of concreteness of the content of the thoughts", and "Indicate the degree of abstractness of the content of the thoughts") using a horizontal line $(0-100 \mathrm{~mm})$, with at the left end of the continuum " $0 \% /$ neutral", and at the right end " $100 \% "$.
Quantitative Manipulation Check (Self-Rated VAS)

VAS were used to examine the degree to which adolescents had had thoughts according to the instructions during the experimental period. Various aspects of the cognitive coping strategies were measured by several scales per experimental condition. Participants could indicate the degree to which they thought in a certain way about the stressful event during the task on a horizontal line $(0-100 \mathrm{~mm})$ with at the left end of the continuum "totally not", and at the right end "all the time". All items started with "During the experiment ...". The following items were used to measure the degree of rumination: "I thought about the negative aspects of the event over and over again (e.g., how stressful the event was)"' "I tried to understand what the causes are of the event, over and over again"/ II thought about the consequences of the event over and over again" ( $\alpha=0.64)$. The following item was used to measure the degree of distancing: "I looked at myself/the situation from a distance, as if I were somebody else". The following items were used to measure the degree of positive reappraisal: "I thought about the positive things that I could get out of the event/situation"/"I actually did get positive things out of the situation"/ "I thought about how the event made me stronger" $(\alpha=0.78)$. The following items were used to measure the degree of acceptance: "I tried to deal with my thoughts at great length, without finding them good or bad" "I felt that it gradually became easier to accept my thoughts about the stressful event" $(\alpha=0.48)$. Furthermore, some general items (all VAS) were added, i.e., "When you were thinking just now, did you especially think in words, thus that your thoughts consisted of sentences and words (as if you're talking to yourself?)" and "When you were thinking just now, did you especially think in images, thus as if you see before you what you think?". To check for the potentially constructive effect of a reflective form of rumination we added the following item to the manipulation check: "During the task I came to a better understanding of myself, and of the event".

\section{Brief Overview of Statistical Analyses}

There were no missing data, because questionnaires were administered through a computer-based programme. AN(C) OVAs were conducted to examine the effect of condition on change in affect states and on content of thoughts (manipulation checks). In case the assumption of homogeneity or normality was violated, we carried out Kruskal-Wallis tests with Mann-Whitney tests for pair wise comparisons. Linear regression analyses revealed no influential outliers (i.e., cases with a standardized residual above 3 ). In addition to the examination of statistically significant differences, 
we computed Cohen's $d$ effect sizes to assess the magnitude of the differences between conditions (see APA 2001). By including effect sizes, power analyses and comparisons to effects found in other studies can be made (Wilkinson and the APA Task Force on Statistical Inference 1999). Therefore, ANCOVAs were carried out with dummy-coded variables for condition and $\mathrm{T} 1$ affect state as covariates, and the change score for affect state (T3-T2) as dependent variable. Cohen's $d$ s were also computed for the differences between conditions on the manipulation check items.

\section{Results}

Manipulation Check: General Findings

The stressful events that were reported by the participants were the following: fight with friend or loved one (26.9\%); death of a loved one (22.5\%); divorce or fights between parents $(9.4 \%)$; mental or physical illness of a loved one (7.5\%); disappointing school results (7.5\%); love troubles $(6.9 \%)$; bullying $(5.6 \%)$, and not classifiable $(13.8 \%)$. $X^{2}$-tests revealed no significant differences between the frequencies of event category per condition, meaning that categories of stressful events were equally reported across conditions (information obtainable from first author). To check for demand effects, at the very end of the experiment participants were asked to write down what they thought was the purpose of the current study. Answers showed that the participants appeared unaware of the true purpose of the study. ${ }^{3}$

\section{Qualitative Manipulation Check (Observer-Rated)}

Intraclass correlation coefficients were adequate to high with respect to the observer-rated degrees of rumination, positive reappraisal, distancing, acceptance, self-focus, and concreteness/abstractness of thoughts (ICCs $=0.78-0.98$ ), indicating good to excellent agreement between observers. Therefore, mean rating scores were computed. Significant group differences were found on the mean rating scores for rumination, positive reappraisal, distancing, acceptance, and concreteness of thoughts. Table 1 displays ICCs,

\footnotetext{
$\overline{3}$ They thought that we wanted to examine: "what people feel/think about stressful events" (47.5\%); "influence of thoughts/memories on mood" (15.6\%); "how we cope with thoughts/feelings/events" $(14.4 \%)$; "influence of music on mood" (11.9\%); "don't know" $(10.6 \%) . X^{2}$-tests showed that the frequencies of answers per category did not significantly differ between conditions (information obtainable from first author).
}

descriptives and test statistics. The observers identified significantly more ruminative thinking in the rumination condition (from here referred to as "RUM") compared to the distancing condition (from here referred to as "DIS"; $U-415.00, z=3.77, p<0.001, d=0.39)$, acceptance condition (from here referred to as "ACC"; $U=350.00, z=4.28$, $p<0.001, d=0.44$ ) and positive reappraisal condition (from here referred to as "POS"; $U=241.50, z=5.70, p<0.001$, $d=0.53)$. Significantly more positive reappraisal was rated in POS compared to RUM $(U=307.50, z=5.53, p<0.001$, $d=0.53)$, ACC $(U=324.50, z=5.08, p<0.001, d=0.50)$, and DIS $(U=288.00, z=5.73, p<0.001, d=0.54)$. The degree of distancing was higher in DIS compared to RUM $(U=558.00$, $z=2.48, p=0.01, d=0.27)$ and POS $(U=482.50, z=3.56$, $p<0.001, d=0.37)$; but not ACC $(U=693.00, z=0.88$, $p=0.38, d=0.10)$. The degree of acceptance was rated higher in ACC compared to POS $(U=554.00, z=2.81, p=0.005$, $d=0.30$ ), but did not significantly differ from that in RUM ( $U=628.50, z=1.66, p=0.10, d=0.19)$, and DIS ( $U=652.50$, $z=1.43, p=0.15, d=0.16$ ), the differences however were in the expected direction. In sum, these findings indicate that our manipulations were successful, except for acceptance. Conditions did not differ with regard to the ratings of degree of self-focus and abstractness of the thoughts. Level of concreteness was higher in DIS compared to POS $(U=612.50, z=1.97, p<0.05, d=0.22)$ and ACC $(U=542.00, z=2.35, p<0.05, d=0.26)$, and higher in RUM compared to ACC $(U=578.00, z=1.99, p<0.05, d=0.22)$.

\section{Quantitative Manipulation Check (Self-Report)}

Reliability coefficients of the composite checks for rumination and positive reappraisal were adequate $(\alpha s=0.64$ and 0.78 respectively, see Table 2). Reliability of the acceptance check scale, consisting of only two items, was low $(\alpha=$ 0.48 ). The Spearman-Brown prediction formula indicated a reliability of $\rho=0.82$ should the scale have consisted of 10 items. ANOVAs and Kruskal-Wallis tests showed significant group differences on the manipulation checks for rumination, positive reappraisal, and distancing. Descriptives and statistics are displayed in Table 2. Participants in RUM reported significantly more rumination compared to those in POS, $t(156)=4.00, p=0.001$, $d=0.63$, and ACC, $t(156)=4.00, p=0.001, d=0.63$; whereas the degree of rumination did not significantly differ between RUM and DIS, $t(156)=2.25, p=0.15, d=0.36$; however, the direction of effect is more ruminations in RUM. Participants in POS reported significantly more positive reappraisal than those in RUM $(U=416.50, z=3.81$, $p<0.001, d=0.85)$, ACC $(U=323.00, z=4.59, p<0.001$, $d=1.05)$, and DIS $(U=272.00, z=5.18, p<0.001, d=1.16)$. In DIS, participants reported more distancing than those in RUM ( $U=377.50, z=4.07, p<0.001, d=0.93)$, POS 
Table 1 Differences between conditions on observer-rated thought content: raw means, standard deviations, ICCs, and test statistics

\begin{tabular}{|c|c|c|c|c|c|c|c|}
\hline \multirow[b]{2}{*}{ Variable } & \multicolumn{4}{|l|}{ Condition } & \multirow[b]{2}{*}{$\chi^{2}(d f=3)$} & \multirow[b]{2}{*}{$r$} & \multirow[b]{2}{*}{$I C C$} \\
\hline & $\begin{array}{l}\text { RUM }(N=40) \\
M(S D)\end{array}$ & $\begin{array}{l}\text { DIS }(N=40) \\
M(S D)\end{array}$ & $\begin{array}{l}\operatorname{POS}(N=41) \\
M(S D)\end{array}$ & $\begin{array}{l}\operatorname{ACC}(N=39) \\
M(S D)\end{array}$ & & & \\
\hline Degree of rumination & $64.93(41.98)$ & $31.25(37.66)$ & $11.48(25.68)$ & $25.85(32.70)$ & $39.52 * *$ & 0.77 & 0.90 \\
\hline Degree of distancing & $20.19(32.41)$ & $43.54(41.54)$ & $13.36(31.46)$ & $36.83(38.46)$ & $16.67 * *$ & 0.80 & 0.87 \\
\hline Degree of positive reappraisal & $3.54(16.39)$ & $1.89(7.37)$ & $61.96(45.39)$ & $5.29(17.23)$ & $58.76^{* *}$ & 0.90 & 0.98 \\
\hline Degree of acceptance & $11.96(22.22)$ & $18.06(33.18)$ & $8.84(25.01)$ & $23.38(29.53)$ & $8.02 *$ & 0.66 & 0.80 \\
\hline Degree of self-focus & $61.98(31.69)$ & $56.84(34.81)$ & $69.21(29.67)$ & $55.77(36.69)$ & 3.35 & 0.66 & 0.78 \\
\hline Degree of concreteness of thoughts & $44.35(38.66)$ & $45.92(35.34)$ & $31.30(32.27)$ & $27.91(34.13)$ & $7.96^{*}$ & 0.82 & 0.91 \\
\hline Degree of abstractness of thoughts & $35.50(31.48)$ & $24.79(27.16)$ & $34.10(29.88)$ & $35.37(32.82)$ & 2.52 & 0.72 & 0.85 \\
\hline
\end{tabular}

${ }^{* *} p \leq 0.001,{ }^{*} p<0.05$. Degree of rumination/distancing/positive reappraisal/acceptance="Indicate the degree to which the text fits the rumination/ distancing/positive reappraisal/acceptance condition according to you". Degree of self-focus="To what extent is the subject focused on him/her self?". Degree of concreteness/abstractness of thoughts="Indicate the degree of concreteness/abstractness of the content of the thoughts"

$(U=286.50, z=5.04, p<0.001, d=1.14)$, and ACC $(U=$ $267.00, z=5.03, p<0.001, d=1.16)$. No significant group differences appeared on the acceptance manipulation check, indicating that the manipulation of acceptance did not result in higher numbers of acceptance ratings.

Groups did not significantly differ with respect to selfreported severity of the stressor, generally indicated as "quite severe". No group differences were found regarding the degree to which participants reported to have come to a better understanding of themselves and the situation. The ratio of thinking in words by images significantly differed between conditions, with a higher words/images ratio in POS compared to DIS ( $U=502.00, z=3.00, p<0.005, d=0.32)$ and $\operatorname{ACC}(U=532.50, z=2.57, p=0.01, d=0.28)$.

\section{The Effect of Condition on Change in Negative} and Positive Affect States ${ }^{4}$

Reliability in terms of internal consistency was high for all questionnaires $(\alpha \mathrm{s}=0.86-0.90)$, see Table 3 . The scores on the CDI were not normally distributed and therefore underwent a log linear transformation resulting in acceptable skewness and kurtosis values $(-1$ to +1$)$. One-way ANOVAs showed no pre-existing differences between conditions on the SRRS-C, CDI, and T1 affect states.

\footnotetext{
${ }^{4}$ ANCOVAs were also carried out with level of depressive symptoms (CDI) as a moderator of condition. Results showed that level of depressive symptoms did not influence the effect of condition on the change in affect states; nor did level of depressive symptoms influence the change in affect states after the manipulations. Furthermore, the moderating effect of gender on condition was examined. Results showed that gender did not influence the effect of condition, nor did gender influence the change in affect after the manipulations.
}

Group means and test statistics on all questionnaires and affect measurements (T1, T2, T3) are displayed in Table 3. Change scores were created by subtracting the measurement of mood state before the manipulation of cognitive strategy (T2), from the measurement of mood state after manipulation of cognitive strategy (T3). Then we checked whether the means plots of the change scores for the state variables of gloominess and sadness displayed a similar trend across conditions. Subsequently, the VAS measuring sadness and gloominess were summed up into a composite score labelled "negative affect" $(\alpha=0.63)$. In the ANCOVA model, condition was the fixed factor, with the change score on affect state as the dependent variable, controlling for trait-rumination (SRRS-C), and T1 affect state. To examine whether the effect of condition was dependent on the degree of trait-rumination, the interaction term of condition with the level of trait-rumination was added to the model and removed when not significant.

\section{Effect of Condition on Negative Affect State}

Trait-rumination did not moderate the effect of condition, $F(3,154)=0.42, p=0.74$. The main effect of condition was significant, $F(3,154)=4.13, p=0.008$ (see Table 3 for groups means). Simple contrasts were used for pair wise comparisons, with a False Discovery Rate correction for the number of tests ( $p=0.02$ for 6 tests; Narum 2006). Contrasts showed that the decrease in negative affect was significantly stronger in POS compared to DIS, $t(156)=3.33$, $p<0.001, d=0.53$; POS to RUM, $t(156)=2.37, p=0.019$, $d=0.40$, and POS to ACC, $t(156)=2.49, p=0.014, d=0.40$. RUM did not differ from ACC, $t(156)=0.09, p=0.93$, $d=0.00$, nor DIS, $t(156)=0.94, p=0.35, d=0.15$; and DIS not from ACC $t(156)=0.85, p=0.40, d=0.14$. Cohen's $d \mathrm{~s}$ indicate small to medium differences between POS and the 
Table 2 Differences between conditions on self-rated thought content: raw means, standard deviations, and test statistics

\begin{tabular}{|c|c|c|c|c|c|c|c|}
\hline \multirow[b]{3}{*}{ Variable } & \multirow[b]{3}{*}{$\alpha$} & \multicolumn{4}{|l|}{ Condition } & \multirow{2}{*}{\multicolumn{2}{|c|}{$d f=3$}} \\
\hline & & RUM $(N=40)$ & DIS $(N=40)$ & $\operatorname{POS}(N=41)$ & $\operatorname{ACC}(N=39)$ & & \\
\hline & & $M(S D)$ & $M(S D)$ & $M(S D)$ & $M(S D)$ & $F$ & $\chi^{2}$ \\
\hline Manipulation check rumination & 0.64 & $76.18(17.11)$ & $65.60(23.58)$ & $57.51(21.02)$ & $57.30(21.69)$ & $7.20 * * *$ & \\
\hline Manipulation check distancing & $\mathrm{N} / \mathrm{a}$ & $37.95(33.83)$ & $68.33(19.30)$ & $33.29(29.00)$ & $33.62(27.97)$ & & $33.98 * * *$ \\
\hline Manipulation check pos. reappraisal & 0.78 & $44.19(26.97)$ & $37.59(24.81)$ & $65.99(16.78)$ & $43.80(23.05)$ & & $32.96^{* * *}$ \\
\hline Manipulation check acceptance & 0.48 & $63.19(17.87)$ & $62.51(20.02)$ & $63.89(19.68)$ & $61.79(18.24)$ & 0.09 & \\
\hline Manipulation check reflection & $\mathrm{N} / \mathrm{a}$ & $48.73(30.57)$ & $42.03(24.73)$ & $51.32(23.00)$ & $43.49(24.91)$ & 1.14 & \\
\hline Self-rated severity of the event & $\mathrm{N} / \mathrm{a}$ & $3.88(0.82)$ & $3.95(0.99)$ & $3.83(0.92)$ & $3.67(0.90)$ & 0.69 & \\
\hline Thinking in words & N/a & $52.45(36.50)$ & $41.93(27.96)$ & $57.59(33.46)$ & 39.79 (32.29) & & 7.32 \\
\hline Thinking in images & $\mathrm{N} / \mathrm{a}$ & $58.93(35.58)$ & $76.88(23.28)$ & $48.88(32.51)$ & $66.82(28.05)$ & & $15.36^{* *}$ \\
\hline Ratio words/images & $\mathrm{N} / \mathrm{a}$ & $8.63(23.14)$ & $1.86(7.83)$ & $9.22(23.12)$ & $4.31(16.55)$ & & $10.53^{*}$ \\
\hline
\end{tabular}

$* * * p \leq 0.001, * * p<0.005, * p<0.05$ (ANOVAs for parametric data and Kruskal-Wallis tests for non-parametric data)

other conditions, with greater decreases in negative affect in POS. Covariate stress-reactive rumination did not have a significant influence on the change in negative affect, $F(1,154)=0.04, p=0.84$, nor did $\mathrm{T} 1$ negative affect, $F(1,154)=1.34, p=0.25$. Figure 1a depicts the mean course of negative affect per condition over time.

\section{Effect of Condition on Positive Affect State}

Trait-rumination did not moderate the effect of condition, $F(3,154)=0.51, p=0.68$. The main effect of condition was significant, $F(3,154)=8.68, p<0.001$ (see Table 3 for group means). Simple contrasts showed that the increase in positive affect was significantly stronger in POS compared to RUM, $t(156)=3.39, p=0.001, d=0.54$, POS to ACC, $t(156)=3.15, p=0.002, d=0.50$, and POS to DIS, $t(156)=4.97, p<0.001, d=0.80$, with medium to large effect sizes. Simple contrasts did not reveal any other statistically significant group differences regarding the change in positive affect: RUM did not differ from ACC, $t(156)=0.26, p=0.79, d=0.00$. DIS did not differ from RUM, $t(156)=1.52, p=0.13, d=0.25$, nor from ACC, $t(156)=1.77, p=0.08, d=0.30$. Although statistically not significant, Cohen's $d$ s indicate a small but relevant greater increase in positive affect in RUM and ACC, compared to DIS. Covariate stress-reactive rumination did

Table 3 Group differences on depressive symptoms, trait-rumination, and negative and positive affect states

\begin{tabular}{|c|c|c|c|c|c|c|c|c|}
\hline \multirow[b]{3}{*}{ Variable } & \multirow[b]{3}{*}{$\alpha$} & \multicolumn{4}{|l|}{ Condition } & \multirow{2}{*}{\multicolumn{3}{|c|}{$d f=3,156$}} \\
\hline & & RUM $(N=40)$ & DIS $(N=40)$ & $\operatorname{POS}(N=41)$ & $\operatorname{ACC}(N=39)$ & & & \\
\hline & & $M(S D)$ & $M(S D)$ & $M(S D)$ & $M(S D)$ & $F$ & $\chi^{2}$ & $p$ \\
\hline Depressive symptoms (CDI) & 0.86 & $8.58(6.50)$ & $8.00(5.94)$ & $7.90(5.20)$ & $6.97(5.65)$ & & 3.87 & 0.28 \\
\hline Rumination (SRRS-C) & 0.90 & $19.15(6.38)$ & $17.93(5.44)$ & $16.41(5.06)$ & $16.95(4.30)$ & 2.03 & & 0.11 \\
\hline T1 negative affect & 0.87 & $20.60(26.09)$ & $23.73(23.28)$ & $14.32(17.00)$ & $14.17(18.75)$ & 1.94 & & 0.13 \\
\hline T2 negative affect & 0.85 & $63.86(26.06)$ & $65.31(24.54)$ & $64.35(25.03)$ & $55.55(23.92)$ & 1.30 & & 0.28 \\
\hline T3 negative affect & 0.80 & $56.34(26.51)$ & $63.37(28.60)$ & $43.52(26.27)$ & $47.88(26.80)$ & 4.30 & & 0.006 \\
\hline Change score negative affect & 0.63 & $-7.53(20.96)$ & $-1.94(29.02)$ & $-20.83(23.01)$ & $-7.67(21.03)$ & 4.63 & & 0.004 \\
\hline $\mathrm{T} 1$ positive affect & $\mathrm{N} / \mathrm{a}$ & $70.30(23.62)$ & $67.58(24.96)$ & $71.27(22.14)$ & $75.97(23.91)$ & 0.86 & & 0.46 \\
\hline $\mathrm{T} 2$ positive affect & $\mathrm{N} / \mathrm{a}$ & $31.60(23.59)$ & $30.90(20.58)$ & $32.02(26.77)$ & $41.82(26.39)$ & 1.76 & & 0.16 \\
\hline T3 positive affect & $\mathrm{N} / \mathrm{a}$ & $36.33(25.19)$ & $28.08(24.69)$ & $52.93(24.41)$ & $47.51(26.11)$ & 7.96 & & 0.001 \\
\hline Change score positive affect & $\mathrm{N} / \mathrm{a}$ & $4.73(20.05)$ & $-2.83(19.36)$ & $20.90(25.51)$ & $5.69(20.09)$ & 8.75 & & 0.001 \\
\hline
\end{tabular}

Raw (untransformed) means are reported. CDI=Children's Depression Inventory; SRRS-C=Stress-reactive Rumination Scale for Children; Negative affect=composite gloomy and sad (VAS); Positive affect=happy (VAS). T1 =baseline; T2=pre-manipulation; T3=post-manipulation 
Fig. 1 a Mean course of negative affect state per condition. T1-T2: Stress Induction; T2-T3: Cognitive Strategy; T3-T4: Positive Mood Induction. b Mean course of positive affect state per condition. T1-T2:

Stress Induction; T2-T3: Cognitive Strategy; T3-T4: Positive Mood Induction
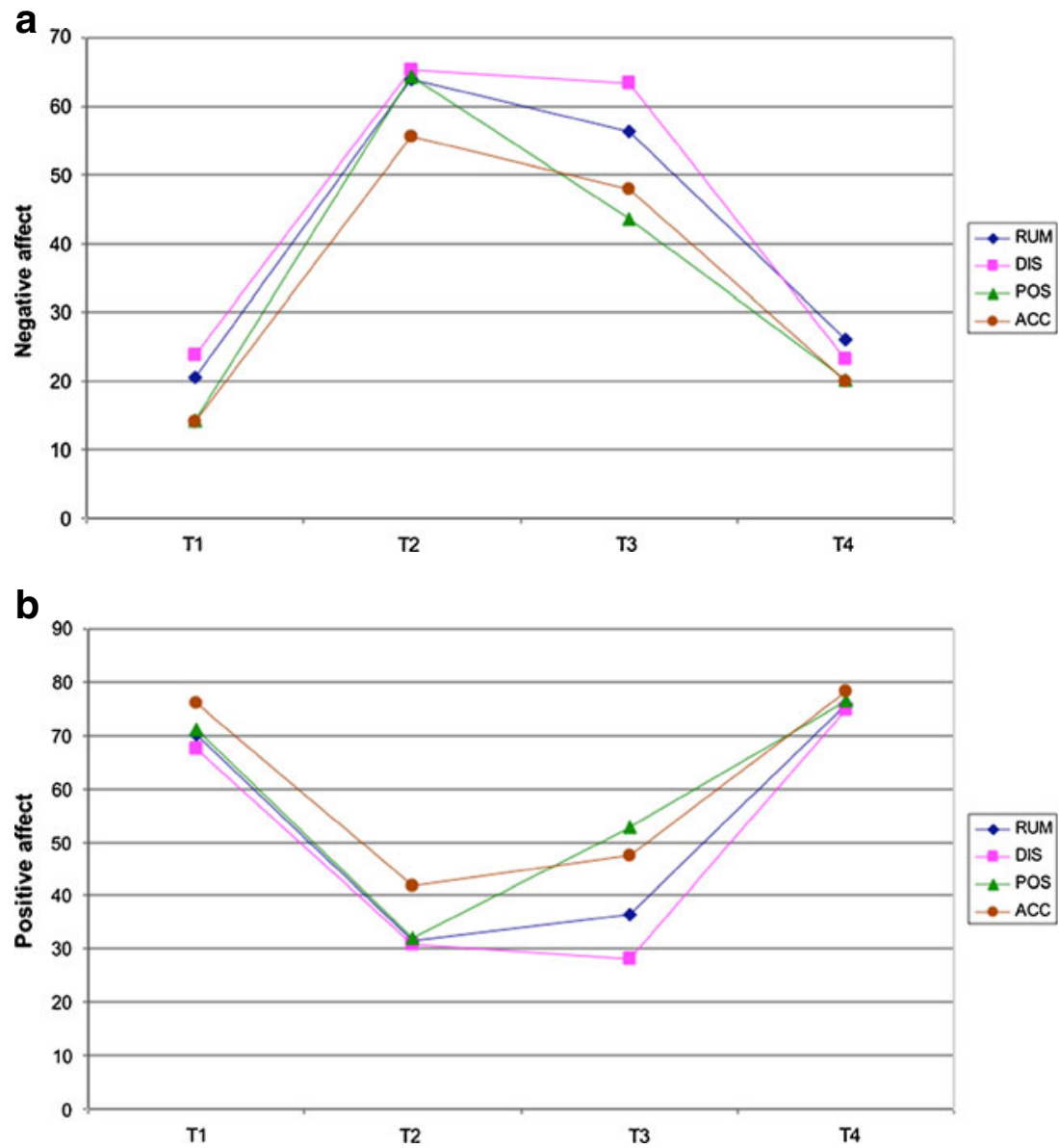

not have a significant influence on the change in positive affect, $F(1,154)=0.14, p=0.71$, nor did T1 positive affect, $F(1,154)=0.01, p=0.99$. Figure $1 \mathrm{~b}$ depicts the mean course of positive affect per condition over time.

\section{Discussion}

The aim of the present study was to compare the effects of experimentally induced rumination, acceptance, distancing, and positive reappraisal on negative and positive affect states in a sample of adolescents. In sum, results showed that a brief manipulation of positive reappraisal in response to thinking about a recent stressful experience significantly increased positive affect, and decreased negative affect, compared to rumination, acceptance, and distancing. This means that after thinking about a personal stressor, adolescents' positive affect improved most, and negative affect decreased most, when they thought about what they learned from a stressful experience, and how it made them stronger. These findings are in line with earlier studies demonstrating a positive relationship between positive reappraisal and well-being (see Helgeson et al. 2006), and point to a beneficial role for positive reappraisal in influencing both positive and negative affect.

An interesting finding is that positive reappraisal seemed to be characterized by more verbal activity than imagery compared to the other conditions. The positive words/ images ratio may be indicative of an abstract level of construal, which is intrinsic to meaning-based coping (Folkman 2008), and which has been shown to be constructive in the context of positive mood with respect to motivation and goal setting (Labroo and Patrick 2009; Vasquez and Buehler 2007). As rumination may be a predominantly verbal and abstract activity (McLaughlin et al. 2007; Stöber and Borkovec 2002), positive reappraisal might be an attractive alternative coping strategy for predominantly "verbal" thinkers. However, the long-term effect of positive reappraisal on mood should be examined in order to check whether positive reappraisal is not some form of cognitive avoidance (see Borkovec et al. 1998).

Unexpectedly, the short-term effect of acceptance of thoughts and feelings was not beneficial over the effect of rumination or distancing. This is inconsistent with Broderick (2005) and Singer and Dobson (2007); however, it is consistent with Kuehner et al. (2009), who found that the 
effect of mindful self-focus on mood did not differ from rumination in a non-clinical sample. There are three potential explanations for this null-finding. First, acceptance may be more effective in reducing negative mood states in (formerly) depressed individuals (Kuehner et al. 2009). Second, since groups did not differ on the manipulation checks for acceptance, our acceptance manipulation may have failed. Either the instructions were not effective, or adolescents have not yet developed the cognitive capacity or maturity to follow the acceptance instructions. Third, beneficial effects on mood are more likely to be expected after weeks of mindfulness training in which acceptance is practiced intensively (see Kabat-Zinn 2003; Segal et al. 2002), or perhaps after a longer manipulation; acceptance implicates exposure to all (also negative) aspects of the event, which could explain why the short-term effect of acceptance was not beneficial over rumination.

Remarkable is the unexpected decrease in negative affect in the rumination condition. This finding is not in line with Park et al. (2004), who found that rumination increased depressed mood in non-clinical adolescents. First, it needs to be remarked that there seems to be a ceiling effect for negative affect after the stress-induction, as participants generally reported maximum levels of negative affect at T2, which makes it impossible to have higher rates of negative affect at T3 (post-manipulation). Second, it is possible that paradoxically the effect of naturally occurring rumination was attenuated, because the cards offer a certain degree of structure which would not be present in natural ruminative thinking, or that our instructions elicited also more constructive forms of rumination (see Treynor et al. 2003); however, our manipulation checks showed that participants did not gain more insight after rumination compared to the other conditions. Another explanation could be that rumination in response to stress is not per se maladaptive in non-dysphoric subjects as has been found in adult samples (Nolen-Hoeksema et al. 2008). According to Watkins (2008), level of abstract processing and negative thought content determine whether the outcome of rumination is unconstructive and could lead to worsening of negative mood. It should be noted that the rumination condition was not characterized by reduced concreteness/ increased abstractness of thoughts compared to the other conditions, moreover, the rated levels of abstractness were generally low (perhaps due to the level of cognitive development), which might explain why rumination did not maintain, but slightly improved negative affect. Distancing maintained negative affect state, which is consistent with findings regarding the role of imagery in evoking emotions (see Holmes and Mathews 2010), and with the finding that taking an observer's perspective without engaging in reflection leads to negative affect (Kross et al. 2005).
The effect of condition was not dependent of a general tendency to ruminate. The current finding may implicate that non-clinical adolescents with a relatively stronger tendency to ruminate benefit from applying positive reappraisal when thinking about stressful events as much as other adolescents. However, considering the fact that the mean scores on the SRRS-C in this sample ranged between average (i.e., "sometimes") and below average, and that the standard deviations were relatively low, an alternative explanation for this null-finding may be that there was insufficient variability in trait-rumination scores to be able to compare high and low trait-ruminators. Thus, current questions should be examined in more clinically proximal ways.

As for clinical implications, Garnefski et al. (2002) showed that adults use significantly more positive reappraisal to cope with stressful events compared to adolescents, suggesting that positive reappraisal is a coping strategy that one learns to employ later in life. However, the current study demonstrates that adolescents have the capacity to apply this coping strategy, and that it works for them. Adolescents might benefit from being made aware of this strategy earlier in life by psycho-education; furthermore, positive reappraisal could be applied in intervention and prevention strategies for youth experiencing difficulties with affective functioning.

This study contributes to existing literature on the role of various cognitive coping strategies in the regulation of affect in youth by its experimentally controlled design. As yet, only Park et al. (2004) have examined the effect of rumination on the maintenance of depressed mood in youth experimentally. A strength of the present study is that a self-rated as well as an observer-rated manipulation check showed that our inductions of positive reappraisal, rumination, and distancing (but not acceptance) succeeded. Another strength is that participants were to select a personally relevant topic to cope with. Furthermore, the sample is relatively large and representative of Dutch youth in terms of educational level and SES.

Some limitations need to be addressed as well. First, the manipulations were short and there was no follow-up measurement. Second, we did not choose for a thinkaloud procedure during the cognitive coping manipulation, because we did not want to disturb the thinking process, but checked the effect of the manipulations through a writing assignment afterwards. As a result, the written texts might not represent all thoughts during the experiment. Moreover, we might not have captured the process of thinking, which may be equally important as the content of thoughts. Finally, although checks showed that participants did not guess the full purpose of the study, there is still a possibility that demand effects influenced the results, as we did not check whether they expected our instructions to be helpful.

Future experimental research should examine the longterm effects of rumination, positive reappraisal, acceptance, 
and distancing on the regulation of affect states in adolescents including psycho-physiological measures. It would be interesting to compare the effects of a short training in these cognitive coping strategies on the regulation of positive and negative affect states in response to stress. Furthermore, the effects of coping strategies on third variables, such as problem-solving ability, overgeneral memory, or attentional biases, need examination. Testing the current hypotheses in high risk and clinically depressed youth samples is warranted in order to further shape models of the pathogenesis of depression, as well as intervention and prevention programs. To conclude, when thinking about stressful events, positive reappraisal significantly enhances positive affect and decreases negative affect in the short-term, compared to rumination, acceptance, and distancing.

Open Access This article is distributed under the terms of the Creative Commons Attribution Noncommercial License which permits any noncommercial use, distribution, and reproduction in any medium, provided the original author(s) and source are credited.

\section{Appendix: Instruction Manipulation of Cognitive Coping Strategy}

"You just thought of a stressful event. You are expected to think about that event again, but now in a certain way*: Now try to think about the causes of that stressful event over and over again, about the consequences, and about how the event moves you. ${ }^{1}$ Now try to think about the positive sides of the stressful event. Examine what you have learned, and how it has made you stronger. ${ }^{2}$ Now try to allow all thoughts about the stressful event, without thinking that a thought is bad or good. Accept that you have them, and see the thought as only a thought. Try to notice that your thoughts come and go. ${ }^{3} /$ Now try to describe the stressful event for yourself from beginning to end, but from a distance, as if you are a journalist, or a filmmaker, or a photographer. (You may) just think about the negative aspects of the event! ${ }^{4}$ You have been given cards by the researcher, which can help you get going. Use these cards to think about the stressful event(s). You may decide yourself how much time you spend with one card, as long as you try to answer the question on the card for yourself. If you have had all the cards, just pick the first card again and try to answer it again, but this time even more precisely. Of course it can be the case that you have to think about several stressful things. You may decide yourself whether you think about one event, or whether you bring up several events. Try to keep to the instructions! Try not to go and think about something else than the stressful events, even if that can be pretty difficult! Some people think in words, others see images before them. You may decide yourself whether you think in words or in images. The researcher will leave you alone with your thoughts for a while, and indicate when you can stop. Mind! After the experiment is over, we will ask you to write down on the computer what went through your mind (thoughts and images)."

*Note. Specific instructions were added for each condition: ${ }^{1}$ Rumination; ${ }^{2}$ Positive reappraisal; ${ }^{3}$ Acceptance; ${ }^{4}$ Distancing.

\section{Examples of Cards}

Rumination: "Think about what the causes are of the event/ situation"

Positive reappraisal: "Think about how this event has finally made you stronger"

Acceptance: "Notice that your thoughts come and go" Distancing: "Think about what is said and thought by every 'actor' that is present on the set, and how [it is said and thought]"

\section{References}

American Psychological Association (APA). (2001). Publication manual of the American Psychological Association (5th ed.). Washington, DC: Author.

Baer, R. A. (2003). Mindfulness training as a clinical intervention: a conceptual and empirical review. Clinical Psychology: Science and Practice, 10(2), 125-143.

Biegel, G. M., Brown, K. W., Shapiro, S. L., \& Schubert, C. M. (2009). Mindfulness-Based Stress Reduction for the treatment of adolescent psychiatric outpatients: a randomized clinical trial. Journal of Consulting and Clinical Psychology, 77(5), $855-866$.

Bögels, S. M., \& Mansell, W. (2004). Attention processes in the maintenance and treatment of social phobia: hypervigilance, avoidance and self-focused attention. Clinical Psychology Review, $24,827-856$.

Bögels, S. M., Hoogstad, B., van Dun, L., de Schutter, S., \& Restifo, K. (2008). Mindfulness training for adolescents with externalizing disorders and their parents. Behavioural and Cognitive Psychotherapy, 36, 193-209.

Borkovec, T. D., Ray, W. J., \& Stöber, J. (1998). Worry: a cognitive phenomenon intimately linked to affective, physiological, and interpersonal behavioral processes. Cognitive Therapy and Research, 22(6), 561-576.

Broderick, P. C. (2005). Mindfulness and coping with dysphoric mood: contrasts with rumination and distraction. Cognitive Therapy and Research, 29(5), 501-510.

Burke, C. A. (2010). Mindfulness-based approaches with children and adolescents: a preliminary review of current research in an emergent field. Journal of Child and Family Studies, 19, 133-144.

Compas, B. E., Connor-Smith, J. K., Saltzman, H., Harding Thomsen, A., \& Wadsworth, M. E. (2001). Coping with stress during childhood and adolescence: problems, progress, and potential in theory and research. Psychological Bulletin, 127, 87-127. 
Folkman, S. (2008). The case for positive emotions in the stress process. Anxiety, Stress, and Coping, 21(1), 3-14.

Folkman, S., \& Moskowitz, J. T. (2000). Positive affect and the other side of coping. American Psychologist, 55(6), 647-654.

Fredrickson, B. L. (2004). The broaden-and-build theory of positive emotions. Philosophical Transactions of the Royal Society of London: Biological Sciences, 359, 1367-1377.

Garnefski, N., Kraaij, V., \& Spinhoven, P. (2001). Negative life events, cognitive emotion regulation and emotional problems. Personality and Individual Differences, 30, 1311-1327.

Garnefski, N., Legerstee, J., Kraaij, V., van den Kommer, T., \& Teerds, J. (2002). Cognitive coping strategies and symptoms of depression and anxiety: a comparison between adolescents and adults. Journal of Adolescence, 25, 603-611.

Germer, C. K. (2005). Mindfulness: What is it? What does it matter? In C. K. Germer, R. D. Siegel, \& P. R. Fulton (Eds.), Mindfulness and psychotherapy (pp. 3-27). New York: The Guilford.

Gross, J. J. (2002). Emotion-regulation: affective, cognitive, and social consequences. Psychophysiology, 39, 281-291.

Heeren, A., \& Philippot, P. (2009). Changes in ruminative thinking mediate the clinical benefits of mindfulness. Poster session presented at the 39th Annual Meeting of the European Association for Behavioral and Cognitive Therapies, Dubrovnik, Croatia.

Helgeson, V. S., Reynolds, K. A., \& Tomich, P. L. (2006). A metaanalytic review of benefit finding and growth. Journal of Consulting and Clinical Psychology, 74(5), 797-816.

Holmes, E. A., \& Mathews, A. (2010). Mental imagery in emotion and emotional disorders. Clinical Psychology Review, 30, 349-362.

Huffziger, S., \& Kuehner, C. (2009). Rumination, distraction, and mindful self-focus in depressed patients. Behaviour Research and Therapy, 47, 224-230.

Kabat-Zinn, J. (2003). Mindfulness-based interventions in context: past, present, and future. Clinical Psychology: Science and Practice, 10(2), 144-156.

Kenny, L. M., \& Bryant, R. A. (2007). Keeping memories at an arm's length: vantage point of trauma memories. Behaviour Research and Therapy, 45, 1915-1920.

Kovacs, M. (1981). Rating scales to assess depression in school-aged children. Acta Paedopsychiatrica, 46, 305-315.

Kross, E., \& Ayduk, Ö. (2009). Boundary conditions and buffering effects: does depressive symptomatology moderate the effectiveness of self-distancing for facilitating adaptive emotional analysis? Journal of Research in Personality, 43, 923-927.

Kross, E., Ayduk, Ö., \& Mischel, W. (2005). When asking "why” does not hurt: distinguishing rumination from reflective processing of negative emotions. Psychological Science, 16(9), 709-715.

Kuehner, C., Huffziger, S., \& Liebsch, K. (2009). Rumination, distraction, and mindful self-focus: effects on mood, dysfunctional attitudes and cortisol response. Psychological Medicine, 39, 219-228.

Labroo, A. A., \& Patrick, V. M. (2009). Psychological distancing: why happiness helps you see the big picture. Journal of Consumer Research, 35, 800-809.

McLaughlin, K. A., Borkovec, T. D., \& Sibrava, N. J. (2007). The effects of worry and rumination on affect states and cognitive activity. Behavior Therapy, 38, 23-38.

Mor, N., \& Winquist, J. (2002). Self-focused attention and negative affect: a meta-analysis. Psychological Bulletin, 128, 638-662.

Narum, S. R. (2006). Beyond Bonferroni: Less conservative analyses for conservation genetics. Conservation Genetics, 7, 783-787.

Nigro, G., \& Neisser, U. (1983). Point of view in personal memories. Cognitive Psychology, 15, 467-482.

Nolen-Hoeksema, S. (1987). Sex differences in unipolar depression: evidence and theory. Psychological Bulletin, 101(2), 259-282.
Nolen-Hoeksema, S. (1991). Responses to depression and their effects on the duration of depressive episodes. Journal of Abnormal Psychology, 100(4), 569-582.

Nolen-Hoeksema, S., Wisco, B. E., \& Lyubomirsky, S. (2008). Rethinking rumination. Perspectives on Psychological Science, $3,400-424$.

Park, R. J., Goodyer, I. M., \& Teasdale, J. D. (2004). Effects of induced rumination and distraction on mood and overgeneral autobiographical memory in adolescent Major Depressive Disorder and controls. Jounal of Child Psychology and Psychiatry, 45 (5), 996-1006.

Robinson, M. S. (1997). The role of negative inferential style and stress-reactive rumination on negative inferences in the etiology of depression: Empirical investigation and clinical implications. Unpublished doctoral dissertation, Temple University.

Robinson, M. S., \& Alloy, L. B. (2003). Negative cognitive styles and stress-reactive rumination interact to predict depression: a prospective study. Cognitive Therapy and Research, 27(3), 275292.

Rood, L., Roelofs, J., Bögels, S. M., Nolen-Hoeksema, S., \& Schouten, E. (2009). The influence of emotion-focused rumination and distraction on depressive symptoms in non-clinical youth: a meta-analytic review. Clinical Psychology Review, 29, 607-616.

Rood, L., Roelofs, J., Bögels, S. M., \& Alloy, L. B. (2010). Dimensions of negative thinking and the relations with symptoms of depression and anxiety in children and adolescents. Cognitive Therapy and Research, 34, 333-342.

Segal, Z. V., Williams, J. M. G., \& Teasdale, J. D. (2002). Mindfulnessbased cognitive therapy for depression. New York: The Guilford.

Singer, A. R., \& Dobson, K. S. (2007). An experimental investigation of the cognitive vulnerability to depression. Behaviour Research and Therapy, 45, 563-575.

Stöber, J., \& Borkovec, T. D. (2002). Reduced concreteness of worry in generalized anxiety disorder: findings from a therapy study. Cognitive Therapy and Research, 26(1), 89-96.

Timbremont, B., \& Braet, C. (2001). Psychometrische evaluatie van de Nederlandstalige Children's Depression Inventory. Gedragstherapie, 34(3), 229-242.

Timbremont, B., \& Braet, C. (2002). Children's depression inventory: Nederlandstalige versie [Children's Depression Inventory: Dutch version]. Lisse: Swets \& Zeitlinger.

Treynor, W., Gonzalez, R., \& Nolen-Hoeksema, S. (2003). Rumination reconsidered: a psychometric analysis. Cognitive Therapy and Research, 27(3), 247-259.

Tugade, M. M., \& Fredrickson, B. L. (2004). Resilient individuals use positive emotions to bounce back from negative emotional experiences. Journal of Personality and Social Psychology, 86 (2), 320-333.

Vasquez, N. A., \& Buehler, R. (2007). Seeing future success: does imagery perspective influence achievement motivation? Personality and Social Psychology Bulletin, 33, 1392-1405.

Watkins, E. (2008). Constructive and unconstructive repetitive thoughts. Psychological Bulletin, 134, 163-206.

Watson, D., Clark, L. A., \& Carey, G. (1988). Positive and negative affectivity and their relation to anxiety and depressive disorders. Journal of Abnormal Psychology, 97, 346-333.

Wilkinson, L., \& the APA Task Force on Statistical Inference. (1999). Statistical methods in psychology journals: guidelines and explanations. American Psychologist, 54, 594-604.

Williams, M., Teasdale, J. D., Segal, Z., \& Kabat-Zinn, J. (2007). The mindful way through depression: freeing yourself from chronic unhappiness. New York: The Guilford. 